\section{Manipulation of inflammation in ARDS: achievable goal or distant target?}

\section{S V Baudouin}

\section{A possible role for thioredoxin}

$\mathrm{T}$ he acute respiratory distress syndrome (ARDS) was only recognised as a distinct clinical entity less than 50 years ago. ${ }^{1}$ It arose as a consequence of the success of modern resuscitation and organ support and was first described in severely injured military personnel. It is defined by the development of rapidly progressing respiratory failure, usually within 24-48 hours of the initiating insult. Plain chest radiographs show widespread airspace shadowing with a pattern similar to cardiogenic pulmonary oedema However, when measured, left sided cardiac pressures are normal and the pulmonary oedema fluid has a high protein content.

This picture of normal cardiac filling pressures and high air space protein content suggested that the central pathophysiology was a result of increased pulmonary epithelial/ endothelial permeability. Histological examination of biopsy and autopsy specimens showed widespread epithelial and endothelial damage and radioisotope studies confirmed that lung permeability was abnormal.

Histological studies also indicated that an intense inflammatory response was occurring in the lungs of patients with established ARDS. ${ }^{23}$ In addition to proteinaceous material, the air spaces were filled with acute inflammatory cells-predominantly neutrophils and macrophages. This early phase of ARDS has been characterised as the "exudative phase". Limited studies suggest that this may be rapidly followed by the proliferation of type II pneumocytes with the beginnings of alveolar basement repair. Fibrosis is also a feature of this phase with the migration of fibroblasts and myoblasts into the fibrinous intra-alveolar exudate. A subsequent fibrotic phase can then occur with extensive lung remodelling, and this may produce a situation of irreversible end stage lung disease. It must be emphasised, however, that this orderly pattern is based on very limited biopsy material and many patients with ARDS make a very good functional recovery over a prolonged period of time.
The theme of lung inflammation has been taken up in this issue of Thorax by Callister and co-workers who measured the concentration of thioredoxin in patients with established ARDS. ${ }^{4}$ The alveolar spaces of the lung are uniquely accessible to investigators and BAL is a well established and safe investigation, even in patients with severe lung injury. Callister and his collaborators found that BAL fluid concentrations of thioredoxin were higher in ARDS patients than in healthy volunteers. BAL fluid concentrations of thioredoxin were also significantly higher than plasma levels, suggesting that thioredoxin was being locally released into the alveolar space. This view was supported by immunohistochemistry findings of raised thioredoxin in pulmonary macrophages and alveolar type II epithelial cells.

\section{THIOREDOXINS}

The thioredoxins (Trx) are a family of evolutionary conserved, small molecular weight thiol proteins. ${ }^{5-7}$ They exist in several forms, with the cytosolic located Trx- 1 and the mitochondria based Trx-2 being the most prevalent. Interest in these molecules has gathered pace in the last few years and it is now well established that they play a key role in the regulation of reduction-oxidation (redox) balance. They fulfil this function in a complex and multifaceted manner. From a biochemical perspective, they transfer reducing equivalents to disulfide groups in target proteins. They are then reduced back to the dithiol form by an NADPH-dependent flavoprotein, thioredoxin reductase. This group constitutes the so-called Trx system.

Several in vitro and in vivo studies indicate that thioredoxin is responsive to cell stresses that alter redox balance. Following cell stress and activation, thioredoxin translocates to the nucleus; there is also significant extracellular release of thioredoxin. In man, increased circulating levels of thioredoxin have been reported in several conditions including heart failure, asthma, acute myocardial infarction, bronchoalveolar lavage (BAL) fluid of severe burns, and type II diabetes mellitus. Thioredoxin concentrations in BAL fluid were also raised in patients with sarcoidosis.

Thioredoxin is involved in several key areas of inflammation and cellular repair. The apparent multiplicity of its in vitro actions prevents any simple categorisation as either a "pro-inflammatory" or "anti-inflammatory" agent. It has anti-oxidant properties and acts as a co-factor to maintain the peroxiredoxin (Prx) family of proteins in reduced form. These, in turn, inactivate hydrogen peroxide. However, physiological concentrations of thioredoxin are much lower than other anti-oxidant systems (such as the glutathione pathway) and a direct anti-oxidant role may not occur in vitro.

It is likely that the major role of the Trx system lies in the regulation of signal transduction and gene regulation. $^{5-7}$ Thioredoxin has anti-apoptotic actions. Exogenous thioredoxin prevents oxidant induced apoptosis in some cell lines and transfected cells that overexpress Trx-1 are resistant to apoptosis. The precise signal transduction pathways responsible for these effects remain unknown, but may involve thioredoxin binding with transcription factors including apoptosis signal regulating kinase 1 (ASK-1). The converse effect of this anti-apoptopic action is growth promotion. This is exemplified by the observation that Trx-1 and Trx-2 null mice die at an embryonic stage of development.

Thioredoxin also has multiple actions on the inflammatory pathways. Both induction and inhibition of cytokine release have been reported. These actions may also be mediated by transcription factor activation with evidence for Trx- 1 activation of NF- $\kappa \beta$ and other factors involved in the regulation of the innate immune response. Other relevant actions are a direct in vitro chemoattractant effect on monocytes, neutrophils, and $\mathrm{T}$ cells. However, in other reports, intravenous thioredoxin reduced lipopolysaccharide induced neutrophil chemotaxis.

These complex properties of thioredoxin underscore a major difficulty facing researchers in ARDS who have tried to translate the many studies of inflammatory mediators into clinically effective treatment. There is no doubt that ARDS is an inflammatory condition, and the paper by Callister et al further confirms and extends these observations. However, several well conducted randomised controlled trials of various anti-inflammatory agents and anti-oxidants have failed to show an improved outcome in ARDS. ${ }^{8}$ 
A number of explanations are possible:

- Disease heterogeneity.

- Complexity of the inflammatory response.

- Timing of the intervention.

- Inflammation may be beneficial.

- The inflammatory stimulus is still present.

\section{Disease heterogeneity}

Patients recruited into most trials fulfil a simple clinical criterion of reduced gas exchange and abnormal radiology. Neither the presence of increased lung permeability nor inflammation is included in the clinical trial definition of ARDS. It is perhaps not surprising that anti-inflammatory agents may fail into such a broad grouping. The current paper also highlights the fact that different patient subsets may have different inflammatory responses. The study divided patients into those with direct and indirect lung injury and found higher inflammatory marker levels in those with direct injury. Further sub-divisions could be proposed-such as pneumonia versus aspiration-and each of these may show significant variation in the inflammatory response. The "one anti-inflammatory fits all" hypothesis may therefore be flawed.

\section{Complexity of the inflammatory response}

Micro-array techniques are beginning to indicate the complexity of the inflammatory response. ${ }^{9}$ Many hundreds of genes are simultaneously upregulated and downregulated and this is reflected in the seemingly endless discovery of "new" mediators. The reductionist approach has made it difficult for clinical researchers to focus on key inflammatory events. In this respect, molecules like thioredoxin do appear to fulfil many of the criteria for "major player". However, clinical researchers in ARDS may still rightly wonder if they are "lost in translation".

\section{Timing of the intervention}

Experimental studies of ALI indicate that early treatment with anti-inflammatory agents, preferably before the insult, is most likely to be effective. In practice this would only be applicable in a few situations. However, the current study and other reports indicate that inflammation persists in ARDS. The possibility of later rescue therapy cannot therefore be dismissed.

\section{Inflammation may be beneficial}

The inflammatory and repair pathways are not separate and distinct. ${ }^{10}$ Teleologicaly, they both carry out the same function of promoting recovery from injurious agents. The complex actions of thioredoxin demonstrate the difficulty in separating out advantageous from disadvantageous actions. Both thioredoxin antagonists and recombinant thioredoxin are available and could be subjected to clinical trials in ARDS. The basic science would support both approaches. Thioredoxin has a number of pro-inflammatory actions so an antagonist trial seems attractive. However, an equally compelling case could be made for a replacement study based on the hypothesis that it is lack of thioredoxin that prevents the resolution of lung injury.

\section{The inflammatory stimulus is still present}

Experimental studies which began in the 1970s strongly suggested that mechanical ventilation causes lung injury-so-called ventilator-induced lung injury (VILI). ${ }^{11}$ Even with modest tidal volumes and airway pressures, all animal species studied developed a pattern of lung injury which was indistinguishable from human ARDS. More recent studies in man have confirmed that ventilation can exacerbate lung injury in ARDS. Indirect support has come from trials of low versus higher tidal volumes in ARDS. ${ }^{12}$ The largest of these studies, conducted by the ARDSnet, reported significantly worse outcomes in patients receiving the higher tidal volume/airway pressure regime. In addition, a number of studies have reported the presence of higher concentrations of pro-inflammatory mediators in BAL fluid samples from ARDS patients ventilated with higher versus lower tidal volumes. The lack of effect of anti-inflammatory therapy in ARDS may be a result of our inability to remove the primary driving stimulus to continuing lung injury-that is, ventilation. It is, of course, possible that specific pathways in the VILI process will be amenable to targeted treatment. However, current research indicates that these pathways will be as complicated as those which underlie the initial lung injury process.

\section{CONCLUSIONS}

It is now well established that an intense inflammatory response occurs in the air spaces in ARDS. Whether this is the primary damaging stimulus or a vital repair process remains undecided, and probably these two effects cannot be separated. Inflammation remains an attractive therapeutic target in ARDS. However, the key pathways involved are proving to be highly complex and not simply divisible into pro- and antiinflammatory limbs. In the light of this increasing knowledge, it is perhaps not surprising that previous trials of antiinflammatory agents in ARDS have failed.

Thorax 2006;61:464-465.

doi: 10.1136/thx.2005.057265

Correspondence to: Dr S V Baudouin, Senior Lecturer in Critical Care Medicine, Royal Victoria Infirmary, Newcastle upon Tyne, UK; s.v.baudouin@ncl.ac.uk

Competing interests: none declared.

\section{REFERENCES}

1 Ware LB, Matthay MA. The acute respiratory distress syndrome. N Engl J Med 2000;342:1334-49.

2 Matthay MA, Zimmerman GA. Acute lung injury and the acute respiratory distress syndrome: four decades of inquiry into pathogenesis and rational management. Am J Respir Cell Mol Biol 2005:33:319-27.

3 Puneet P, Moochhala S, Bhatia M. Chemokines in acute respiratory distress syndrome. Am J Physiol Lung Cell Mol Physiol 2005;288:L3-15.

4 Callister ME, Burke-Gaffney A, Quinlan GJ, et al. Extracellular thioredoxin levels are increased in patients with acute lung injury. Thorax 2006;61:521-7.

5 Watson WH, Yang X, Choi YE, et al. Thioredoxin and its role in toxicology. Toxicol Sci 2004;78:3-14.

6 Winyard PG, Moody CJ, Jacob C. Oxidative activation of antioxidant defence. Trends Biochem Sci 2005;30:453-61.

7 Burke-Gaffney A, Callister ME, Nakamura H Thioredoxin: friend or foe in human disease? Trends Pharmacol Sci 2005;26:398-404.

8 Adhikari N, Burns KE, Meade MO. Pharmacologic therapies for adults with acute lung injury and acute respiratory distress syndrome. Cochrane Database Syst Rev 2004; (4):CD004477

9 Calvano SE, Xiao W, Richards DR, et al. A network-based analysis of systemic inflammation in humans. Nature 2005;437:1032-7.

10 Shimabukuro DW, Sawa T, Gropper MA. Injury and repair in lung and airways. Crit Care Med 2003;31(8 Suppl):S524-31.

11 Lionetti V, Recchia FA, Ranieri VM. Overview of ventilator-induced lung injury mechanisms. Curr Opin Crit Care 2005;11:82-6.

12 Fan E, Needham DM, Stewart TE. Ventilatory management of acute lung injury and acute respiratory distress syndrome. JAMA 2005;294:2889-96. 